\title{
A SEGURANÇA DO PACIENTE EM ÂMBITO HOSPITALAR: REVISÃO INTEGRATIVA DA LITERATURA
}

\author{
Andréia Cristina Araújo Silva ${ }^{1}$, Jéssica Fontinele da Silva ${ }^{1}$, Lidyane Rodrigues Oliveira Santos ${ }^{2}$, Fernanda \\ Valéria Silva Dantas Avelino ${ }^{3}$, Ana Maria Ribeiro dos Santos ${ }^{3}$, Antônio Francisco Machado Pereira ${ }^{4}$
}

\begin{abstract}
RESUMO: Revisão integrativa com o objetivo de identificar as principais temáticas abordadas em ambiente hospitalar para a segurança do paciente. A busca ocorreu em três bases de dados eletrônicas, abrangendo o período de 2009 a 2015, com os descritores: "Segurança do Paciente", "Controle de risco" e "Hospitalização". A amostra do estudo constituiu-se de 34 artigos. Houve aumento significativo no número de publicações sobre a temática em 2013, mais de $100 \%$ comparando-se ao ano de 2009. Dos estudos, $22,22 \%$ tratavam sobre eventos adversos e 18,52\% sobre erros na administração de medicamentos. Dentre as medidas preventivas, 37,93\% destacam a educação continuada e $13,79 \%$ a lavagem das mãos e a identificação correta do paciente. A opção estratégica pela segurança tem sido incluída nas agendas das instituições, organizações e sistemas de saúde como prioridade. Logo, sugere-se ampliar o foco da pesquisa para a melhoria na qualidade do cuidado.
\end{abstract}

DESCRITORES: Segurança do paciente; Controle de risco; Hospitalização.

\section{PATIENT SAFETY IN THE HOSPITAL CONTEXT: AN INTEGRATIVE LITERATURE REVIEW}

\begin{abstract}
This integrative review aimed to identify the main patient safety issues addressed in the hospital context. The search occurred in three electronic databases, covering the period 2009 to 2015, with the following descriptors: "Patient safety", "Risk control" and "Hospitalization". The study sample consisted of 34 articles. There was a significant increase in the number of publications on the subject in 2013 , more than $100 \%$ compared to the year 2009 . Of the studies, $22.22 \%$ dealt with adverse events and $18.52 \%$ with errors in the administration of medication. Among preventive measures, $37.93 \%$ highlighted continuing education and $13.79 \%$ handwashing and the correct identification of the patient. The strategic option for safety has been included in the agendas of health institutions, organizations and systems as a priority. Therefore, it is suggested to expand the focus of research to improve the quality of care. DESCRIPTORS: Patient safety; Risk control; Hospitalization.
\end{abstract}

\section{LA SEGURIDAD DEL PACIENTE EN ÁMBITO HOSPITALAR: REVISIÓN INTEGRATIVA DE LA LITERATURA}

RESUMEN: Revisión integrativa cuyo objetivo fue identificar las principales temáticas abarcadas por el ambiente hospitalar para la seguridad del paciente. La búsqueda ocurrió en tres bases de datos electrónicas, en el periodo de 2009 a 2015, con los siguientes descriptores: "Seguridad del Paciente", "Control de Riesgo" y "Hospitalización". La muestra del estudio fue constituida por 34 artículos. Hubo aumento significativo en el número de publicaciones acerca de la temática en 2013, más de 100\% comparándose al año de 2009. De los estudios, 22,22\% tenían como tema eventos adversos y 18,52\%, errores en la administración de medicamentos. Entre las medidas preventivas, $37,93 \%$ destacan la educación continuada y $13,79 \%$, la higiene de las manos y la identificación correcta del paciente. La opción estratégica por la seguridad viene siendo incluida en las agendas de las instituciones, organizaciones e sistemas de salud como prioridad. Así, se sugiere ampliar el foco de la investigación para perfeccionar la cualidad del cuidado. DESCRIPTORES: Seguridad del paciente; Control de riesgo; Hospitalización.

${ }^{1}$ Enfermeira. Universidade Federal do Piauí. Teresina, PI, Brasil.

${ }^{2}$ Enfermeira. Mestre em Enfermagem. Docente do Instituto Camillo Filho. Teresina, PI, Brasil.

${ }^{3}$ Enfermeira. Doutora em Enfermagem. Docente do Programa de Pós-Graduação em Enfermagem da Universidade Federal do Piauí. Teresina, PI, Brasil.

${ }^{4}$ Enfermeiro. Mestre em Enfermagem. Docente de Enfermagem da Universidade Federal do Piauí. Teresina, PI, Brasil.

Autor Correspondente:

Lidyane Rodrigues Oliveira Santos

Faculdade Santo Agostinho

Av. Presidente Janio Quadros,1165 - 64053-390 - Teresina, Piauí, Brasil

E-mail: lidyanero@yahoo.com.br
Recebido: $27 / 11 / 2014$

Finalizado: 02/06/2016 
As questões associadas à segurança do paciente constituem um problema de saúde em todo o mundo. Nesse sentido, os riscos e a ocorrência de eventos que provocam danos ao paciente têm aumentado em todos os ambientes, em especial em âmbito hospitalar. Nas últimas décadas, nota-se uma preocupação em torno das políticas para a melhoria da qualidade assistencial, e a problemática que envolve os riscos à segurança do paciente se torna tema de vários estudos no sistema de saúde.

A segurança do paciente é definida como o ato de evitar, prevenir ou melhorar os resultados adversos ou lesões originadas no processo de atendimento médico-hospitalar e domiciliar ${ }^{(1)}$. O tema é um grave problema de saúde pública global. Dados apontam que, em países desenvolvidos, um em cada 10 pacientes é prejudicado ao receber cuidados hospitalares. O risco de infecção associado aos cuidados de saúde em alguns países em desenvolvimento é de até 20 vezes maior que em países desenvolvidos ${ }^{(2)}$.

Embora o cuidado em saúde traga enormes benefícios a todos os envolvidos, a ocorrência de erros é possível, e os pacientes podem sofrer graves consequências. Florence Nightingale já colocava que "Pode parecer talvez estranho um princípio enunciar como primeiro dever de um hospital não causar mal ao paciente" (3:34). Florence foi pioneira na prevenção de eventos adversos. Tal prevenção trata-se de um princípio que se torna cada vez mais relevante e atual ${ }^{(2)}$.

Essa temática se tornou foco de atenção no final da década de 90, por meio de publicações sucessivas, dentre elas o livro "To err is human: building a safer health system", elaborado pelo Comitê para a Qualidade do Cuidado à Saúde na América (Committee on Quality of Health Care in America) do: Institute of Medicine of the National Academies (IOM), concluído em 1999 e publicado em 2000 ${ }^{(2)}$.

A partir de então, em 2002, a Organização Mundial de Saúde (OMS), com o apoio dos países membros, lançou a iniciativa de discutir a problemática. Em 2004, por meio do programa da Aliança Mundial para Segurança do Paciente, foram criadas diretrizes e estratégias para incentivar e divulgar práticas que garantissem a segurança do paciente. O referido programa definiu como prioritário o desenvolvimento de pesquisas baseadas em evidências científicas com melhores práticas, bem como iniciativas de pesquisas de maior impacto, que visam instituir medidas que aumentem a segurança do paciente e a qualidade dos serviços de saúde, fomentado pelo comprometimento político dos estados signatários ${ }^{(4)}$.

No Brasil, para a efetivação das metas estabelecidas, iniciou-se monitorização de eventos adversos em 192 hospitais da Rede Sentinela, uma estratégia criada pela Vigilância Sanitária, que passou a funcionar como observatório nos serviços para o gerenciamento de riscos à saúde. Ademais, em 2013, instituiu-se o Programa Nacional de Segurança do Paciente (PNSP), com o objetivo de contribuir para a qualificação do cuidado em saúde em todos os estabelecimentos de saúde no território nacional, para implantar protocolos, núcleos de segurança dos pacientes e notificação de eventos adversos ${ }^{(2,4)}$.

O programa propõe seis protocolos de segurança do paciente com foco nos problemas de maior incidência: cirurgia segura, queda, úlcera por pressão, administração segura de medicação, higiene das mãos e identificação dos pacientes ${ }^{(2)}$.

Embora programas tenham sido implementados para a melhoria na qualidade da assistência, um estudo que abordasse as principais práticas utilizadas em âmbito hospitalar era necessário, em virtude da necessidade de destacar a sua efetivação para a segurança do paciente. Assim, por ser um tema de extrema relevância e atual para ser estudado na perspectiva de melhoria na qualidade da assistência, este estudo teve como objetivo identificar as principais temáticas exploradas, nos anos de 2009 a 2015, assim como medidas preventivas no ambiente hospitalar para a segurança do paciente, com vistas a promover estratégias, ações e sensibilização dos profissionais que atuam no cuidado.

\section{- MÉTODO}

Trata-se de uma revisão de literatura integrativa baseada na pesquisa bibliográfica, método que reúne a produção científica relevante acerca de determinado tema, oferecendo acesso rápido e 
sintetizado aos resultados científicos de maior importância para a área estudada, formulada por meio de artigos publicados sobre as práticas utilizadas para a segurança do paciente(5-6).

Foi realizado um levantamento bibliográfico na Universidade Federal do Piauí, que dispõe de acesso livre a diversos periódicos, no período de outubro a dezembro de 2015, os quais são indexados às bases de dados eletrônicas: LILACS (Literatura Latino-Americana e do Caribe em Ciências da Saúde), SciELO (Scientific Eletronic Library Online) e BDENF (Bancos de Dados da Enfermagem), utilizando os seguintes descritores integrados: Segurança do Paciente/Patient Safety/Seguridad del Paciente, Controle de Risco/Risk Management/Control de Riesgo , Hospitalização/Hospitalization/Hospitalización.

Os critérios de inclusão foram pautados no acesso aos artigos publicados em periódicos nacionais e internacionais, na íntegra em português, inglês e espanhol, de acordo com os descritores, que trouxessem abordagem plena e/ou parcial do objeto de estudo e tivessem sido publicados no período de 2009 a 2015. Assim, foram excluídos os artigos de revisão de literatura, relatos de experiência, artigos reflexivos, editorial, estudo de caso e artigos repetidos.

Foram encontrados 558 artigos nos bancos de dados, sendo 310 no LILACS, 180 no SciELO, 68 no BDENF. Depois da aplicação dos supracitados critérios de exclusão, 76 artigos foram pré-selecionados. Após análise dos resumos, resultados e conclusões, selecionaram-se 34 artigos que atendiam aos objetivos da pesquisa, por meio de formulário pré-estabelecido.

Os dados foram processados, organizados e armazenados em uma planilha eletrônica no programa Excel $^{\circledR}$ (Office $2010^{\circledR}$ ), separados por ano, autor e assunto. Para análise dos dados, foram usados números percentuais para distribuição de frequências por ano, principais autores e temáticas abordadas. Em seguida, os dados foram discutidos à luz da literatura pertinente e demonstrados em gráficos de barras e setores.

A pesquisa abrange a análise das principais temáticas abordadas entre os bancos de dados sobre segurança do paciente, as medidas preventivas para segurança do paciente, além da quantidade de artigos publicados por ano.

\section{RESULTADOS}

A análise dos resultados mostrou, estatisticamente, que houve um significante aumento de publicações sobre as temáticas: segurança do paciente e medidas preventivas encontradas para melhorar a perspectiva de segurança.

O Gráfico 1 mostra a quantidade de artigos encontrados por ano e um crescente aumento de artigos publicados nos últimos anos, como pode ser observado no ano de 2013, que contemplou 10 artigos dos selecionados, ao ser comparado com o ano de 2009, que mostrou apenas quatro estudos.

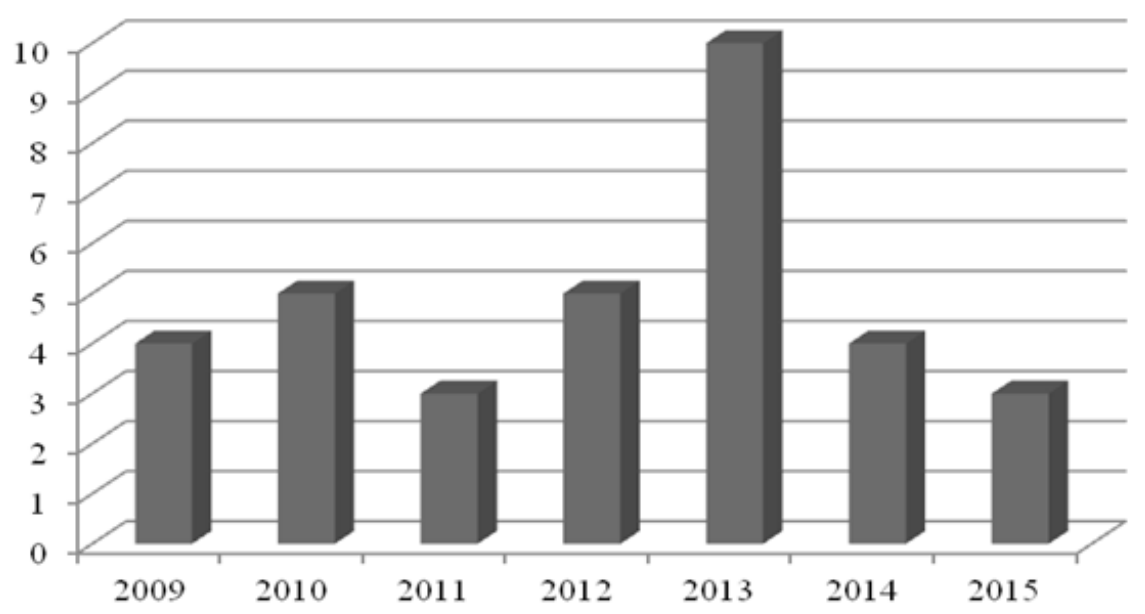

Gráfico 1 - Quantidade de artigos encontrados por ano n=34. Teresina - PI, Brasil 2015 
No Gráfico 2, apontam-se os principais temas nos artigos selecionados sobre segurança do paciente, sendo eventos adversos o tema mais abordado, seguido de erros na administração de medicamentos, lavagem das mãos e educação do profissional. Os demais, apesar de pouco mencionados, mostram-se relevantes para a segurança do paciente.

O Gráfico 3 destaca que, na prevenção de eventos adversos para segurança do paciente, a educação continuada dos profissionais possui lugar de destaque nos artigos selecionados. Na sequência, "Lavagem das mãos", "Identificação correta dos pacientes", "Notificação dos eventos adversos" e "Comunicação efetiva" também aparecem como medidas preventivas.

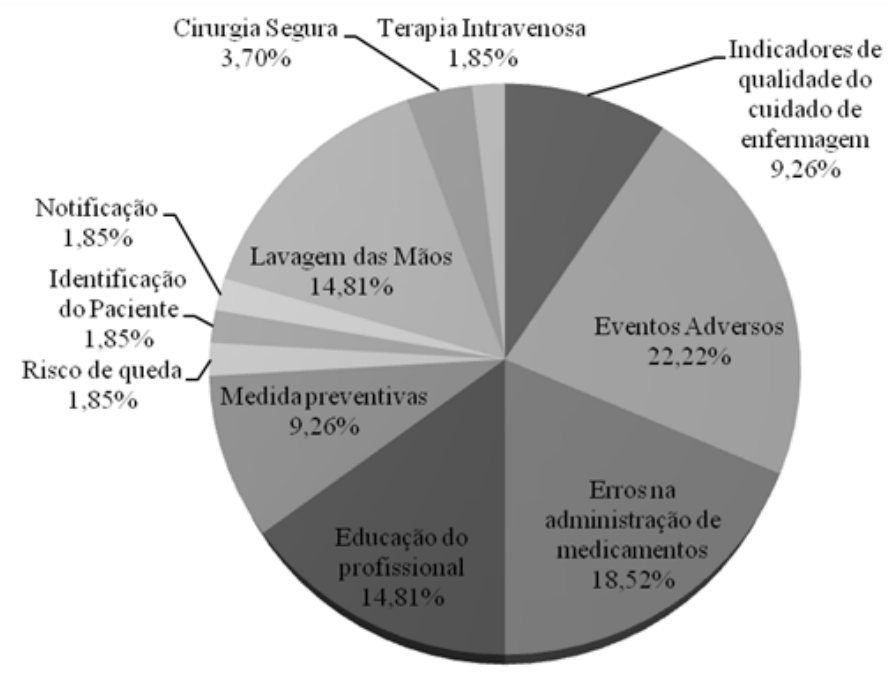

Gráfico 2 - Principais temáticas abordadas sobre segurança do paciente. Teresina-PI, Brasil, 2015

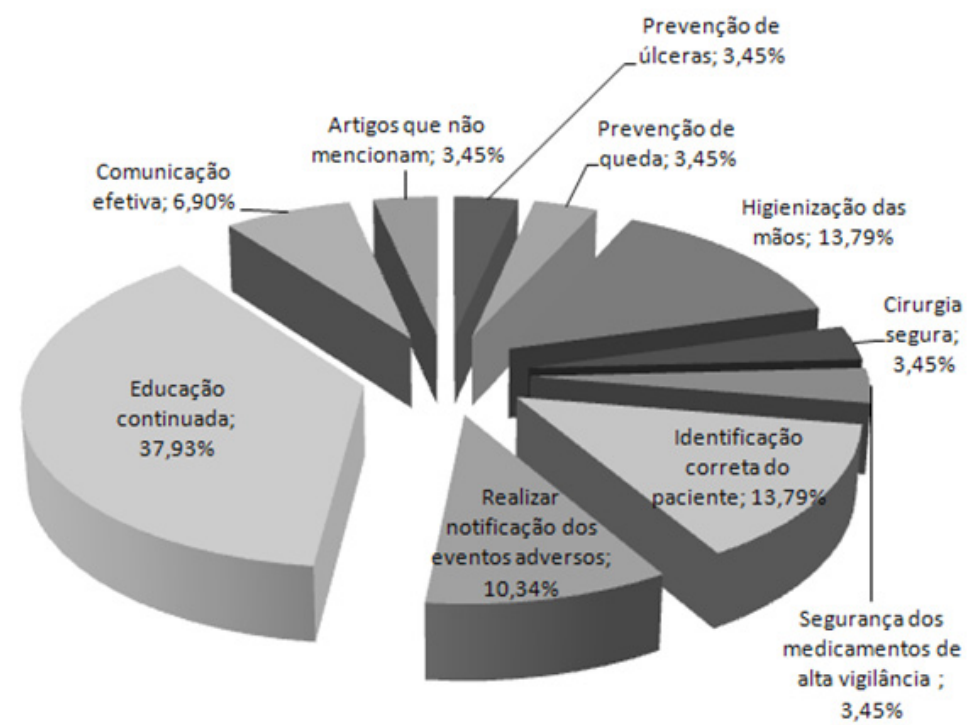

Gráfico 3 - Prevenção de eventos adversos para segurança do paciente. Teresina-PI, Brasil, 2015

\section{DISCUSSÃO}

Os dados mostraram um aumento significativo da produção científica sobre a temática desde o ano de 2009, o que está diretamente relacionado ao maior destaque dado pelos órgãos nacionais e internacionais às políticas de qualidade do cuidado prestado ao usuário na última década.

Em âmbito global, o movimento em prol da segurança do paciente teve seu início na última década do século XX, após a publicação do relatório do Institute of Medicine dos EUA, que apresentou resultados de vários estudos em que se revelou a crítica situação da assistência à saúde naquele país. 
Dados apontaram que, de 33,6 milhões de internações, 44.000 a 98.000 pacientes, aproximadamente, morreram em consequência de eventos adversos ${ }^{(7)}$.

Desde então, a OMS tem demonstrado sua preocupação com a segurança do paciente e adotou essa questão como tema de alta prioridade na agenda de políticas de seus países membros. Em maio de 2007, foram publicadas as nove soluções para prevenção de evento adverso no cuidado à saúde, o que explica esse discreto número de publicações no ano de 2009, tendo em vista que é uma temática nova para pesquisa.

Os dados permitiram analisar as principais temáticas abordadas com vistas a definir a magnitude da problemática. Dentre os achados, os eventos adversos foram os mais relacionados, com um percentual de $22,22 \%$. Nesse contexto, apesar dos avanços na área de saúde, a segurança do paciente ainda é influenciada pelos profissionais de saúde por meio da ocorrência de erros que se refletem diretamente na qualidade de vida dos pacientes. Esses erros denominados "eventos adversos" são caracterizados como ocorrências indesejáveis, porém preveníveis, de natureza prejudicial, que comprometem a segurança do paciente que se encontra sob os cuidados dos profissionais de saúde $^{(7-9)}$.

Dentre os eventos adversos mais citados pelos estudiosos, os erros de dose são um dos problemas mais frequentes relacionados à administração de medicamentos $(18,52 \%)$, interferindo na qualidade da assistência prestada aos pacientes hospitalizados. Esse risco é aumentado na medida em que os profissionais de saúde não são capazes de ler corretamente as prescrições, resultando em confusão durante a dispensação, distribuição, preparo e administração. Por conseguinte, um dos fatores que podem auxiliar na prevenção desses eventos é a correta identificação do paciente, uma vez que clientes com nomes semelhantes, em mesma enfermaria ou recebendo o mesmo medicamento, porém com doses diferenciadas, podem ser facilmente confundidos, passando a receber uma dose inadequada para o seu tratamento ${ }^{(10-14)}$.

Nesse viés, um estudo realizado sobre fatores de risco para ocorrência de erro no preparo de medicamentos intravenosos aponta que os erros mais comuns estão relacionados à preparação de medicamentos e justifica que tal fato ocorre devido à sobrecarga de trabalho e à falta de atenção. Aponta ainda que o preparo antecipado, a reconstituição e diluição inadequadas, ausência de desinfecção das ampolas e bancadas e a omissão da etapa de higienização das mãos foram os principais fatores de risco identificados nessa categoria ${ }^{(15)}$.

Outro estudo relacionado à administração de medicamentos intravenosos em um hospital da Rede Sentinela enfatiza a necessidade de que profissionais de saúde verifiquem a identificação dos pacientes antes da realização de qualquer procedimento. A utilização de placas de identificação em locais visíveis à equipe, e de pulseiras de identificação, também constitui uma importante medida de prevenção de erros na administração de medicamentos ${ }^{(15-16)}$. Considerando outra literatura estudada, ratifica-se a importância do estabelecimento de critérios para padronizar os dados e a linguagem impressa nas etiquetas de identificação ${ }^{(17)}, 13,79 \%$ da amostra mencionam a identificação correta do paciente como medida de prevenção.

Outra temática bastante abordada nos artigos foi a questão da educação profissional, que aparece em $14,81 \%$ dos artigos e é também indicada como a melhor forma de prevenir eventos adversos $(37,93 \%)$. Fato facilmente compreendido, já que o gerenciamento de riscos é trabalho complexo e incorpora diferentes aspectos inerentes à prática profissional, tornando a qualificação da assistência multiprofissional relevante ${ }^{(18-20)}$.

Pesquisadores apontam como desafios nas instituições de saúde para a segurança do paciente: a grande pressão assistencial que os profissionais sofrem, a intensa demanda e a forte carga de trabalho, que dificultam a procura de evidências científicas e novas tecnologias, que se tornam inacessíveis para muitos profissionais. Isso impede a detecção de complicações que, se utilizadas, otimizariam o tempo e produziriam uma consequente melhoria na qualidade da assistência ${ }^{(21-22)}$.

A lavagem das mãos, discutida em 14,81\% dos artigos, surge de forma paralela a outros eventos adversos, pois a sua prática depende principalmente de fatores como educação profissional e corrobora diretamente para diminuição de infecções em ambiente hospitalar. Como fator preventivo isolado, é considerada um fator relevante, com $13,79 \%$. No cotidiano assistencial, contribui para a simplificação de etapas, para agilizar o trabalho e promover a rotinização de oportunidades perdidas para a higienização 
das mãos, prática muitas vezes negligenciada na prioridade das atividades do cuidado ${ }^{(17,23)}$. Diferentes estratégias podem ser empregadas na unidade para promover a adesão à higienização das mãos, tais como feedback dos profissionais, incentivo ao uso de soluções alcoólicas e o estabelecimento de um plano de metas, com o envolvimento de líderes e equipe ${ }^{(19)}$.

Apesar de discreta, a utilização da nomenclatura, a notificação dos eventos adversos e incidentes foi discutida em 1,85\% da amostra estudada. Como fator de prevenção, observou-se em 10,34\% que, além de contribuir para a construção de um banco de dados sobre riscos e situações-problema, também permite a execução de modificações necessárias no processo da assistência, o que promove um processo de trabalho mais seguro e previne futuros eventos adversos ${ }^{(13,20-21)}$.

Frente à notificação de eventos adversos e queixas técnicas realizadas por profissionais de saúde e considerando os resultados que a literatura referencia sobre os acometimentos que acarretam ao paciente, acredita-se que o levantamento e a análise dessas ocorrências possibilitarão o desenvolvimento de programas de educação continuada que estimulem o aumento das notificações e garantam a segurança dos pacientes, resultando em melhoria contínua da qualidade dos serviços de saúde ${ }^{(8,21)}$.

A literatura vigente apoia a necessidade contínua de investigação, notificação e análises das ocorrências que auxiliem no planejamento de intervenções proativas, com a construção de barreiras de defesa para a prevenção de eventos adversos, capazes de proporcionar uma assistência de qualidade e livre de danos aos pacientes ${ }^{(22-24)}$.

Quanto a essa cultura de segurança e comunicação de erros, aparece claramente a falta de comunicação em 6,90\% dos estudos, isso também é descrito devido à dificuldade para confessar o erro. Embora o erro seja inerente ao ser humano, há o temor de punições e, com isso, ocorrem as subnotificações. Dessa forma, surge a necessidade de adaptação às normas legais, trabalhar de forma intensa a segurança clínica das organizações sanitárias, uso de registros adequados e conhecer os erros atuais, o que exige mudança de pensamento e da própria cultura ${ }^{(23-25)}$.

Aponta-se ainda o risco de queda de leito dos pacientes internados em âmbito hospitalar, 1,85\% dos artigos encontrados. Esse risco, na maioria das vezes, está associado a pacientes idosos, do sexo masculino e com doenças neu rológicas, sendo diretamente relacionado ao quantitativo de profissionais nos serviços de saúde e acompanhantes desinformados sobre a condição do paciente. Nesse aspecto, os estudos apontaram a manutenção das grades levantadas e as orientações para os acompanhantes $(9,26 \%)$, como medidas preventivas eficazes ${ }^{(13,25-26)}$.

Ainda, em 1,85\% dos artigos selecionados não são abordados temas específicos sobre as medidas preventivas de segurança do paciente, porém tais artigos tratam da necessidade de se trabalhar as equipes sobre o erro e a culpa como alternativa para modificar e transformar esse acontecimento em oportunidade de discutir e desenvolver o pensar crítico sobre as ações de cuidado e as atitudes frente ao próprio erro e ao do colega. Tudo isso com vistas a perceber o erro como uma oportunidade de aprendizado para impedir novos eventos relacionados à mesma causa ${ }^{(26)}$. Desse modo, é imprescindível conhecer a realidade do trabalho, estimular a participação das pessoas e empregar ferramentas destinadas a gerenciar os riscos, visando minimizar as ocorrências de eventos adversos ou agravos aos usuários dos serviços de saúde ${ }^{(15,17)}$.

A análise evidencia uma visão fragmentada sobre a segurança do paciente nesse ambiente, sendo vista como responsabilidade de uma categoria profissional (médica ou de enfermagem). Assim, propõem-se, de um lado, as afirmativas que apontam certo empoderamento da equipe de enfermagem. Por outro lado, revela-se que a responsabilidade pela segurança não é compartilhada igualmente por todas as equipes ${ }^{(27)}$.

Entretanto, os artigos tratam ou pelo menos citam o Programa de Acreditação Internacional da Joint Commission International (JCl), criado em 1998 e que já acreditou mais de 70 hospitais em 15 países. A acreditação internacional é uma das ferramentas disponíveis para avaliação e padronização da qualidade assistencial prestada por organizadores de saúde. Tal programa trabalha com seis metas: a identificação correta dos pacientes; melhorada comunicação efetiva; segurança de medicamentos de alta vigilância; garantia de cirurgias com local de intervenção correto, procedimento correto e paciente correto; redução de risco de infecções associadas aos cuidados de saúde; e redução dos riscos de 
lesões ao paciente, decorrentes de quedas, colaborando com os resultados levantados na pesquisa ${ }^{(28-32)}$.

Nessa perspectiva, verifica-se uma preocupação das instituições internacionais e nacionais com a política de segurança do paciente, fato esse confirmado com o crescente aumento de publicações sobre a temática de prevenção de eventos ou agravos à segurança do paciente. Tal problemática sugere padrões a serem aplicados nas instituições de saúde para melhorar a assistência ao cliente, a qual exigirá uma longa jornada e que não será fácil nem rápido atender a todas as metas propostas pelo programa de acreditação em muitos hospitais brasileiros. Tal fato torna imprescindível o estabelecimento de um plano de ação coerente e passível de execução, com opções para correção das "não conformidades", planejamento de quantas dessas opções serão postas em prática a cada ano e de taxas esperadas de avanço na avaliação ${ }^{(28-34)}$.

\section{CONCLUSÃo}

Nos últimos anos, tem-se assistido ao pleno desenvolvimento de políticas e estratégias globais em países com diferentes níveis de desenvolvimento, implantadas pela Organização Mundial da Saúde. Dessa maneira, a opção estratégica pela segurança tem sido incluída nas agendas das instituições, organizações e sistemas de saúde.

Os resultados apresentados por este estudo revelam a crescente preocupação das instituições de saúde com o bem-estar dos pacientes e com a melhoria da qualidade do cuidado. No entanto, nos estudos, a avaliação dessa melhoria é realizada por meio de coleta de dados em prontuários, por meio de um desenho de coorte retrospectivo.

A análise permitiu ressaltar que, apesar de ser uma temática bastante discutida atualmente, há que se pesquisar de forma a atender aos anseios dos profissionais sobre métodos adequados de conduzir e melhorar a qualidade da assistência prestada por meio do controle e prevenção dos eventos adversos, com vistas a alcançar uma melhor avaliação da qualidade do cuidado.

\section{REFERÊNCIAS}

1. Rigobello MCG, de Carvalho REFL, Cassiani SHB, Galon T, Capucho HC, de Deus NN . Clima de segurança do paciente: percepção dos profissionais de enfermagem. Acta Paul Enferm. [Internet] 2012; 25(5) [acesso em 08 fev 2014]. Disponível: http://dx.doi.org/10.1590/S0103-21002012000500013

2. Barros CG. Segurança do paciente como prioridade nas organizações hospitalares. [Apresentação do Hospital Albert Einstein; 2013; São Paulo].

3. Pedreira MLG. Enfermagem para a segurança do paciente. Acta Paul Enferm. [Internet] 2009; 22(4) [acesso em 10 fev 2014]. Disponível: http://www.scielo.br/pdf/ape/v22n4/a01v22n4.pdf

4. Ministério da Saúde (BR). Segundo desafio global para a segurança do paciente: orientações para cirurgia segura da OMS. Rio de Janeiro: Ministério da Saúde; 2009.

5. Mendes KDS, Silveira RCCP, Galvão CM. Revisão integrativa: método de pesquisa para a incorporação de evidências na saúde e na enfermagem. Texto contexto-enferm. [Internet] 2008; 17(4) [acesso em 01 jun 2014]. Disponível: http://dx.doi.org/10.1590/S0104-07072008000400018

6. de Souza MT, da Silva MD, de Carvalho R. Revisão integrativa: o que é e como fazer. Einstein. [Internet] 2010; 8(1) [acesso em 01 jun 2014]. Disponível: http://www.astresmetodologias.com/material/O_que_e_RIL.pdf

7. Silva AEBC. Segurança do paciente: desafios para a prática e a investigação em Enfermagem. Rev. Eletr. Enf. [Internet] 2010; 12(3) [acesso em 15 jan 2014]. Disponível: http://dx.doi.org/10.5216/ree.v12i3.11885

8. Bezerra ALQ, e Silva AEBC, Branquinho NCSS, Paranaguá TTB. Análise de queixas técnicas e eventos adversos notificados em um hospital sentinela. Rev. enferm. UERJ. [Internet] 2009; 17(4) [acesso em 20 jan 2014]. Disponível: http://www.facenf.uerj.br/v17n4/v17n4a02.pdf 
9. Mendes W, Pavão ALB, Martins M, Moura MLO, Travassos C. Características de eventos adversos evitáveis em hospitais do Rio de Janeiro. Rev. Assoc. Med. Bras. [Internet] 2013; 59(5) [acesso em 10 jan 2014]. Disponível: http://dx.doi.org/10.1016/j.ramb.2013.03.002

10. Wegner W, Pedro ENR. Patient safety in care circumstances: prevention of adverse events in the hospitalization of children. Rev Latino-am Enfermagem. [Internet] 2012; 20(3) [acesso em 05 fev 2014]. Disponível: http://dx.doi.org/10.1590/S0104-11692012000300002

11. Gimenes FRE, Mota MLSM, Teixeira TCA, Silva AEBC, Opitz SP, Cassiani SHB. Patient safety in drug therapy and the influence of the prescription in dose errors. Rev Latino-am Enferm. [Internet] 2010; 18(6) [acesso em 01 fev 2014]. Disponível: http://dx.doi.org/10.1590/S0104-11692010000600003

12. Corbellini VL, Schilling MCL, Frantz SF, Godinho TG, Urbenetto JS. Eventos adversos relacionados a medicamentos: percepção de técnicos e auxiliares de enfermagem. Rev Bras Enferm. [Internet] 2011; 64(2) [acesso em 13 jan 2014] Disponível: http://www.scielo.br/scielo.php?pid=S0034-71672011000200004\&script=sci_arttext

13. Toffoletto MC, Ruiz XR. Mejorando la seguridad de los pacientes: estudio de los incidentes en los cuidados de enfermería. Rev Esc Enferm USP. [Internet] 2013; 47(5) [acesso em 05 jan 2014]. Disponível: http://dx.doi.org/10.1590/S0080-623420130000500013

14. Lima RPM, Melleiro MM. Percepção da equipe multidisciplinar acerca de fatores intervenientes na ocorrência de eventos adversos em um hospital universitário. Rev Min Enferm. [Internet] 2013; 17(2) [acesso em 20 fev 2014$].$ Disponível: http://www.reme.org.br/artigo/detalhes/652

15. Rodrigues MCS, Oliveira LC. Erros na administração de antibióticos em unidade de terapia intensiva de hospital de ensino. Rev Eletr Enf. [Internet] 2010; 12(3) [acesso em 12 jan 2014]. Disponível:

http://www.revistas.ufg.br/index.php/fen/article/download/11935/7870

16. Camerine FG, Colcher AP, Moraes DS, Souza DL, Vasconcelos JR, Neves RL. Fatores de risco para ocorrência de erro no preparo de medicamentos endovenosos: uma revisão integrativa. Cogitare enferm. [Internet] 2014; 19(2) [acesso em 20 ago 2014]. Disponível: http://ojs.c3sl.ufpr.br/ojs/index.php/cogitare/article/view/37362/22932

17. Camerini FG, da Silva LD. Segurança do paciente: análise do preparo de medicação intravenosa em hospital da rede sentinela.Texto contexto-enferm. [Internet] 2011; 20(1) [acesso em 25 jan 2014]. Disponível:

http://dx.doi.org/10.1590/S0104-07072011000100005

18. Quadrado ERS,Tronchin DMR. Evaluation of the identification protocol for newborns in a private hospital. Rev Latino-am Enferm. [Internet] 2012; 20(4) [acesso em 13 fev 2014]. Disponível:

http://dx.doi.org/10.1590/S0104-11692012000400005

19. Vituri DW, Matsuda LM. Validação de conteúdo de indicadores de qualidade para avaliação do cuidado de enfermagem. Rev Esc Enferm USP. [Internet] 2009; 43(2) [acesso em 15 fev 2014]. Disponível:

http://dx.doi.org/10.1590/S0080-62342009000200024

20. Dias MAE, Martins M, Navarro N. Adverse outcome screening in hospitalizations of the Brazilian Unified Health System. Rev. Saúde Pública. [Internet] 2012; 46(4) [acesso em 30 jan 2014]. Disponível: http://dx.doi. org/10.1590/S0034-89102012005000054

21. Fassini $P$, Hahn GV. Riscos à segurança do paciente em unidade de internação hospitalar: concepções da equipe de enfermagem. Rev Enferm UFSM. [Internet] 2012; 2(2) [acesso em 26 fev 2014]. Disponível: http://cascavel.ufsm.br/revistas/ojs-2.2.2/index.php/reufsm/article/view/4966/3753

22. Ques ÁAM, Montoro CH, Gonzalez MG. Strengths and threats regarding the patient's safety: nursing professionals' opinion. Rev Latino-am Enferm. [Internet] 2010; 18(3) [acesso em 15 jan 2014]. Disponível: http://dx.doi.org/10.1590/S0104-11692010000300007

23. Inoue KC, Matsuda LM, de Melo WA, Murassaki ACY, Hayakawa LY. Risco de queda da cama. O desafio da enfermagem para a segurança do paciente. Invest Educ Enferm. [Internet] 2011; 29(3) [acesso em 26 fev 2014 ]. Disponível: http://www.redalyc.org/articulo.oa?id=105222406015

24. de Sousa MRG, Silva AEBC, Bezerra ALQ, de Freitas JSF, Miasso AI. Eventos adversos em hemodiálise: relatos de profissionais de enfermagem. Rev Esc Enferm USP. [Internet] 2013; 47(1) [acesso em 08 jan 2014]. Disponível: 
25. Capucho HC, Arnas E, Cassiani SHB. Segurança do paciente: comparação entre notificações voluntárias manuscritas e informatizadas sobre incidentes em saúde. Rev. Gaúcha Enferm. [Internet] 2013; 34(1) [acesso em 02 fev 2014]. Disponível: http://dx.doi.org/10.1590/S1983-14472013000100021

26. Bathke J, Cunico PA, Maziero ECS, Cauduro FLF, Sarquis LMM, Cruz EDA. Infraestrutura e adesão à higienização das mãos: desafios à segurança do paciente. Rev. Gaúcha Enferm. [Internet] 2013; 34(2) [acesso em 05 fev 2015$].$ Disponível: http://dx.doi.org/10.1590/S1983-14472013000200010

27. Paese F, Sasso GTMD. Cultura da segurança do paciente na atenção primária à saúde. Texto contexto-enferm. [Internet] 2013; 22(2) [acesso em 17 jan 2014] Disponível: http://dx.doi.org/10.1590/S0104-07072013000200005

28. Bohomol E, Tartali JA. Eventos adversos em pacientes cirúrgicos:conhecimento dos profissionais de enfermagem. Acta Paul Enferm. [Internet] 2013; 26(4) [acesso em 13 fev 2014]. Disponível:

http://dx.doi.org/10.1590/S0103-21002013000400012

29. de Paiva MCMS, de Paiva SAR, Berti HW. Eventos adversos: análise de um instrumento de notificação utilizado no gerenciamento de enfermagem. Rev. esc. Enferm. USP. [Internet] 2010; 44(2) [acesso em 15 de nov 2015 ]. Disponível: http://dx.doi.org/10.1590/S0080-62342010000200007

30. Silva FM, Porto TP, Rocha PK, Lessmann JC, Cabral PFA, Schneider K LK. Higienização das Mãos e a Segurança do Paciente Pediátrico. Cienc. enferm. [Internet] 2013; 19(2) [acesso em 27 out 2015]. Disponível:

http://dx.doi.org/10.4067/S0717-95532013000200010

31. Lorenzini E, Santi JAR, Bao, ACP. Patient safety: analysis of the incidents notified in a hospital, in south of Brazil. Rev. Gaúcha Enferm. [Internet] 2014; 35(2) [acesso em 27 fev 2014]. Disponível:

http://dx.doi.org/10.1590/1983-1447.2014.02.44370

32. Botene DZAB, Pedro ENRB. Os profissionais da saúde e a higienização das mãos: uma questão de segurança do paciente pediátrico. Rev. Gaúcha Enferm. [Internet] 2014; 35(3) [acesso em 15 out 2015]. Disponível: http://dx.doi.org/10.1590/1983-1447.2014.03.44306

33. Françolin L, Gabriel CS, Bernardes A, Silva AEBC, Brito MFPA, Machado JP. Gerenciamento da segurança do paciente sob a ótica dos enfermeiros. Rev. esc. enferm. USP. [Internet] 2015; 49(2) [acesso em 10 nov 2015 ]. Disponivel: http://dx.doi.org/10.1590/S0080-623420150000200013

34. Abreu HCA, Reiners AAL, Azevedo RCS, da Silva AMC, Abreu DROM, de Oliveira AD. Incidência e fatores preditores de quedas de idosos hospitalizados. Rev Saúde Pública. [Internet] 2015; 49(37) [acesso em 10 nov 2015]. Disponível: http://dx.doi.org/10.1590/S0034-8910.2015049005549 